DOI 10. 18307/2017. 0304

(C) 2017 by Journal of Lake Sciences

\title{
巢湖蓝藻聚集对重金属迁移释放的影响”
}

\author{
江 江, 李玉成**, 王 宁, 张学胜 \\ (安徽大学资源与环境工程学院,合肥 230601)
}

\begin{abstract}
摘 要: 以巢湖蓝藻水华聚集地西半湖表层沉积物与蓝藻为对象, 研究了表层沉积物重金属 $(\mathrm{Cd} 、 \mathrm{Cr} 、 \mathrm{Cu} 、 \mathrm{Zn} 、 \mathrm{~Pb} 、 \mathrm{Ni}$ 和 $\mathrm{Mn}$ ) 总量的分布特征. 同时, 利用 BCR 连续提取法对沉积物中重金属 $\mathrm{Cd}$ 和 Mn 的形态进行分析, 模拟探究蓝藻腐殖化过 程中重金属含量的变化, 探讨了蓝藻腐殖化对重金属吸附-释放的影响. 结果表明, 各采样点表层沉积物重金属含量均不 同程度超出背景值, 且距离南汁河越近越突出. 潜在迁移指数表明整个西半湖沉积物中重金属 $\mathrm{Cd}$ 比 Mn 更易形成二次污 染. 相关性分析显示重金属 Cd 和 Mn 各形态含量和总量之间均表现出极显著正相关. 巢湖西半湖属于典型的藻型湖, Cd 和 Mn 的来源和分布均与蓝藻聚集密切相关, 结合实验室模拟蓝藻腐殖化过程对重金属的吸附-释放规律, 认为蓝藻腐殖 化过程会对 $\mathrm{Cd}$ 和 Mn 的迁移释放产生影响.
\end{abstract}

关键词: 巢湖; 重金属; 蓝藻; 腐殖化; 吸附释放

\section{Effect of cyanobacteria aggregation on the migration and release of heavy metals in Lake Chaohu}

JIANG Jiang, LI Yucheng ** , WANG Ning \& ZHANG Xuesheng

( School of Resources and Environmental Engineering, Anhui University, Hefei 230601, P.R. China)

\begin{abstract}
Samples of sediment and cyanobacteria were collected at a cyanobacteria gathering area of western-half area of Lake Chaohu. The distribution patterns of seven heavy metals ( $\mathrm{Cd}, \mathrm{Cr}, \mathrm{Cu}, \mathrm{Zn}, \mathrm{Pb}, \mathrm{Ni}$ and $\mathrm{Mn}$ ) were studied, and the fractions of $\mathrm{Cd}$ and Mn were analyzed using the European Communities Bereau of Reference (BCR) sequential extraction procedure. In addition, laboratorial experiments were carried out to simulate the variations in the heavy metal contents during the cyanobacteria humification, and the effect of cyanobacteria humification on the adsorption and desorption of heavy metals were discussed. The results showed that the heavy metal contents of surface sediments exceed the background values, presenting different values of each sampling site. The closer near the Nanfei River, the higher pollution degree was. Potential Mobile Index analysis showed that, compared to Mn, Cd was more likely to cause the secondary pollution in the western-half area of Lake Chaohu. Correlations analysis indicated that both two heavy metals ( $\mathrm{Cd}$ and $\mathrm{Mn}$ ) showed a significant positive correlation between metal speciation and total metal value. Lake Chaohu was considered as a typical algae lake, and the sources and distributions of two heavy metals ( Cd and Mn) were related to the algae boom. Combined with the study of adsorption and release by cyanobacteria humification, the present study speculates that the cyanobacteria humification could influence the migration and release of $\mathrm{Cd}$ and $\mathrm{Mn}$ in Lake Chaohu.
\end{abstract}

Keywords: Lake Chaohu; heavy metal; cyanobacteria; humification; adsorption and release

因自然或人类活动, 有毒重金属进人水环境, 经过生物降解等途径, 其中不可降解部分最终进人沉积物 中. 湖泊系统中表层沉积物积累的重金属已占生态系统所负担的大部分 ${ }^{[1]}$,使得沉积物重金属污染成为研 究者长期关注的问题之一. 由于重金属存在多种赋存形态, 容易受外界环境条件影响以及与其他物质形成 稳定结合态, 且在外界环境条件发生改变后, 又会与上覆水界面发生物理、化学和生物条件的相互作用,释

* 国家自然科学基金项目 $(41172121,21607001)$ 、安徽省自然科学基金青年项目 (1608085QB45) 和安徽省高等学校 自然科学重点项目 (KJ2015090) 联合资助. 2016-06-25 收稿; 2016-09-21 收修改稿. 江江( 1990 ), 女, 硕士研 究生;E-mail: JJIANG2012anhui@ 126.com.

** 通信作者; E-mail: li-yucheng@163.com. 
放出来造成水体的二次污染, 影响上覆水体的水质 ${ }^{[2-3]}$. 富营养化水体中藻类生物量庞大, 其快速繁殖进行 光合呼吸作用, 使得水体温度、 $\mathrm{pH}$ 、溶解氧等环境理化指标改变, 此外, 在藻类生长过程中分泌大量溶解性有 机质, 死亡机体增加表层沉积物有机质含量, 改变体系的微生物结构等, 这些都对沉积物金属的释放作用产 生复杂的耦合效应 ${ }^{[4]}$.

巢湖 ( $\left.31^{\circ} 25^{\prime} \sim 31^{\circ} 43^{\prime} \mathrm{N}, 117^{\circ} 16^{\prime} \sim 117^{\circ} 5^{\prime} \mathrm{E}\right)$ 位于安徽省中部, 处于长江、淮河两河之间, 是我国五大淡水 湖之一. 水域面积 $769.55 \mathrm{~km}^{2}$, 具有航运、渔业、农灌、防洪等多种功能. 近年来巢湖频繁暴发以铜绿微囊藻 (Microcystis aeruginosa) 为主的蓝藻水华, 尤其是在夏季 6-8 月期间大量繁殖, 西半湖区域内繁殖更加旺 盛 ${ }^{[5-6]}$. 目前对巢湖的研究主要集中在水体的富营养化 ${ }^{[7]}$ 、沉积环境演化 ${ }^{[8-9]}$ 以及巢湖重金属的分布及形态 特征等方面 ${ }^{[10-11]}$. 国内外有关藻类吸附重金属的生物吸附研究已有较全面的研究及综述报道 ${ }^{[12-13]}$, Singh 等 研究发现自然状态下生长的微囊藻比实验室培养的微囊藻具有更强的吸附能力 ${ }^{[14]}$, Rangsayatorn 等研究了 节旋藻 (Arthrospira) 对镉的吸收, 发现藻细胞吸附重金属的最佳 $\mathrm{pH}$ 值为 $7^{[15]}$, 吴文娟等研究了在不同金属 离子浓度和不同 $\mathrm{pH}$ 条件下微囊藻水华对重金属 $\mathrm{Cu}^{2+} 、 \mathrm{Cd}^{2+}$ 和 $\mathrm{Ni}^{2+}$ 的吸附效果 ${ }^{[16]}$.姜晶等测定了蛋白核小球 藻 (Chlorella pyrenoidsa) 对 $\mathrm{Pb}^{2+}$ 和 $\mathrm{Cd}^{2+}$ 的吸附和脱附动力学 ${ }^{[17]}$, 但蓝藻水华及腐殖化过程对巢湖重金属的 迁移释放的影响方面的研究未见报道. 本研究以巢湖西半湖为研究对象, 从蓝藻对重金属的吸附解吸的角 度出发, 对巢湖表层沉积物的总有机碳 (TOC) 、总氮 ( TN) 以及 7 种重金属元素 ( Cd、Cr、Cu、 $\mathrm{Zn} 、 \mathrm{~Pb} 、 \mathrm{Ni}$ 和 $\mathrm{Mn}$ ) 的含量进行测定, 分析其空间分布特征, 并采用 BCR 连续提取法对 Mn 和 Cd 形态指标进行线性相关分 析, 运用潜在迁移指数 $(P M I)^{[18]}$ 评价巢湖西半湖水系的重金属生态风险, 结合 $\mathrm{C} / \mathrm{N}$ 分析重金属的可能来 源, 同时通过模拟实验研究蓝藻腐殖化过程中水体和藻体的重金属含量变化, 验证蓝藻腐殖化过程是否对 重金属有吸附释放效果, 以期认识水华条件下沉积物中重金属的迁移转化规律, 从而为巢湖重金属污染的 治理和环境质量的提升提供指导.

\section{1 材料与方法}

\section{1 样品采集}

于 2013 年 11 月,借助 GPS 全球卫星定位系统, 于巢湖西半湖布设 32 个采样点 (图 1), 使用柱状采样 器采集表层沉积物样品, 装人洁净的聚乙烯自封袋中密封, 低温保存送回实验室进行预处理及后续分析.

\section{2 样品处理}

1.2.1 沉积物样品 采集的样品去掉杂物及石块, 经冷冻干燥后, 经玛瑙研针研磨处理, 过 200 目尼龙篮, 败 存备用. 全湖 32 个采样点 (图 1) 表层沉积物样品用于分析重金属总量、重金属 Cd 与 Mn 形态以及 TOC/TN 比值. 所有样品分析均重复进行 3 次, 以提高精确度和减小随机误差, 试验结果取平均值. 采用欧共体标准 物质局提出的 BCR 连续提取法, 将重金属形态分为弱酸提取态 (F1)、可还原态 (F2)、可氧化态 (F3) 和残渣 态 (F4) ${ }^{[19]}$. 重金属总量和各形态提取液中重金属元素含量采用 $\mathrm{HF}-\mathrm{HClO}_{4}-\mathrm{HNO}_{3}$ ( GR) 混合酸体系消解, 利 用电感耦合等离子体发射光谱 ( ICP-AES) 分析. 为保证分析的准确性, 同步分析了由国家地质实验测试中 心生产的湖底沉积物顺序提取微量元素标准物质(GBW07304a), 各重金属元素不同形态回收率在 $82.3 \%$ $113.1 \%$ 之间, 符合美国国家环境保护局 (USEPA) 标准要求的 $80 \%$ 120\% 的范围. TOC 与 TN 含量采用元素 分析仪 (Vario TOC cube) 测定.

1.2.2 模拟蓝藻腐殖化过程 实验装置采用圆柱形玻璃容器 (图 2), 按 $1: 1$ 比例加入超纯水与新鲜蓝藻, 蓝 藻于 2013 年 7 月取自巢湖西半湖, 正值蓝藻暴发期间, 经镜检 $98 \%$ 以上为蓝藻门微囊藻属 (Microcystis), 蓝 藻藻密度约为 $10^{8}$ cells $/ \mathrm{L}$. 整个实验分成 3 组: $\mathrm{A}$ 组全程好氧; B 组好氧 $30 \mathrm{~d}$, 厌氧 $30 \mathrm{~d}$, 避光处理; $\mathrm{C}$ 组全程 厌氧, 避光处理. 蓝藻反应装置置于 $30 \pm 2^{\circ} \mathrm{C}$ 恒温培养箱中, 不加任何营养元素. 实验阶段, 每隔 $6 \mathrm{~d}$ 取样一 次, 测定上覆水中 $\mathrm{pH}$, 同时测定上覆水与藻体内重金属 $\mathrm{Cd}$ 与 $\mathrm{Mn}$ 含量.

\section{3 数据分析方法}

为定量描述重金属的二次释放污染, 本研究引人潜在迁移指数 (Potential Mobile Index, PMI), 其含义是 指沉积物中某种重金属在环境中可能向其他介质 (如植物、水体等) 迁移的部分占该金属总量的质量百分 比, 即表示迁移能力的强弱. 计算公式为: 


$$
P M I=\frac{B 1+B 2}{\sum B i}
$$

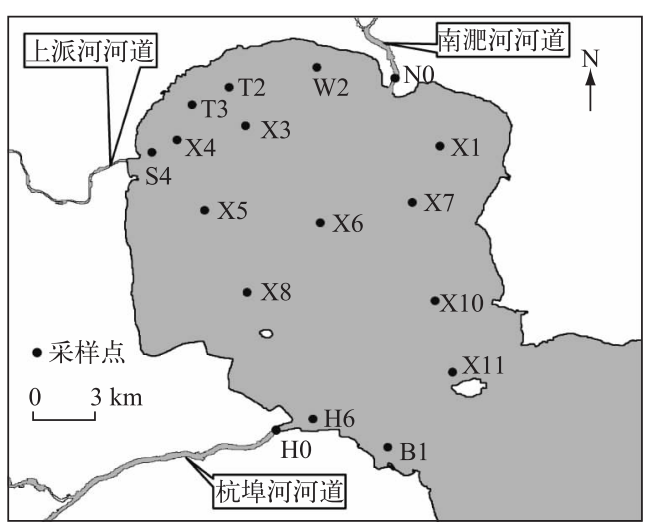

(a)巢湖西半湖

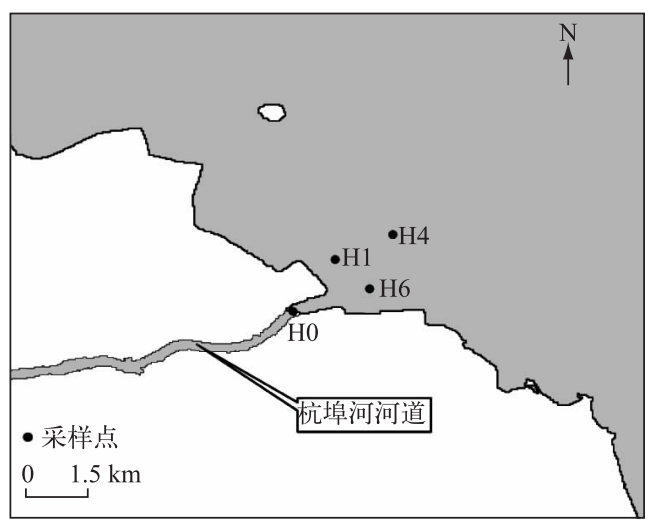

(c)杭埠河

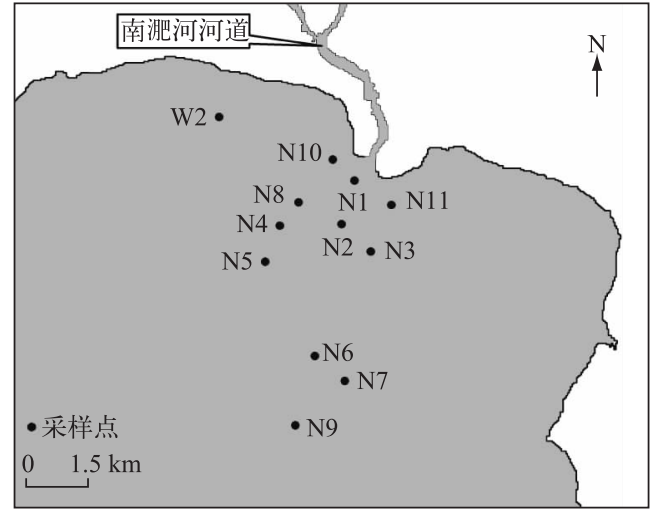

(b)南淝河

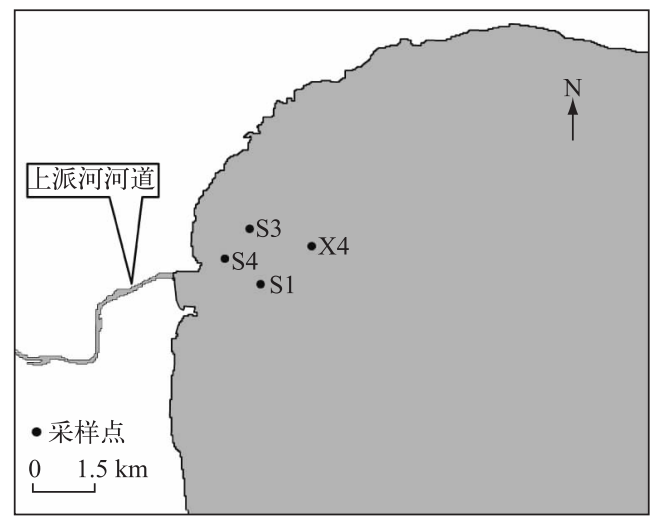

(d)上派河

图 1 巢湖采样点分布

Fig.1 Distribution of sampling sites in Lake Chaohu

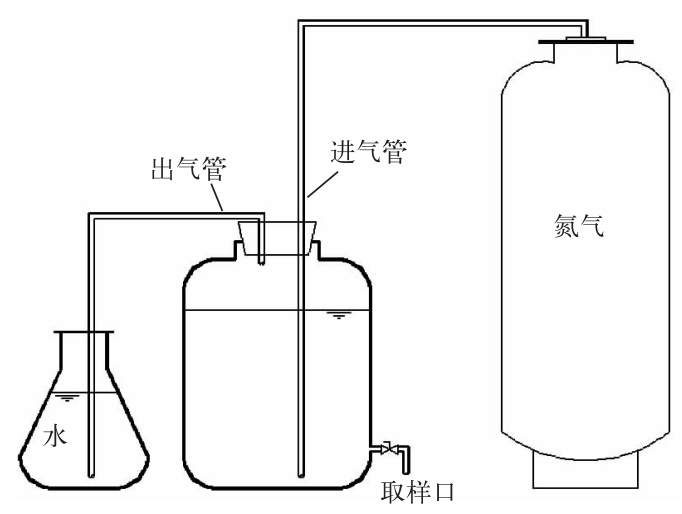

图 2 模拟实验装置

Fig.2 The apparatus of laboratorial experiment
式中, $P M I$ 为某种重金属的潜在迁移指数, 取值 $0 \sim$ $100 \% ; B 1$ 为某种重金属的弱酸提取态的质量分数, $(\mathrm{mg} / \mathrm{kg}) ; B 2$ 为某种重金属的可还原态的质量分数 $(\mathrm{mg} / \mathrm{kg}) ; \sum B i$ 为重金属 4 种形态的质量分数之和, 即该金属在沉积物中的质量分数 $(\mathrm{mg} / \mathrm{kg}) ; i$ 指重金 属 BCR 连续提取中的第 $i$ 种形态, $i=1 、 2 、 3 、 4$.

某种重金属的 $P M I$ 取值越接近 $100 \%$, 则表示该 金属在环境中的潜在迁移性越强 (或活性越强); 相 反, 重金属的 $P M I$ 值越小 (或接近于 0 ), 则表明该金 属在环境中的稳定性越强 (或惰性越强). 这样可以 通过比较重金属的 $P M I$ 值来直观地反映重金属的稳 定度高低.

实验数据采用 Origin 8.5 作图, 采用 SPSS 19.0 软件进行双变量相关性分析, 采用 Pearson 相关系数 分析各组数据之间的相关关系. 


\section{2 结果与讨论}

\section{1 表层沉积物中重金属含量分布特征}

从表 1 可以看出,巢湖各采样点表层沉积物中 7 种重金属含量均不同程度超出背景值 ${ }^{[20]}, \mathrm{Cd} 、 \mathrm{Mn} 、 \mathrm{~Pb}$ 和 $\mathrm{Zn}$ 的含量较背景值普遍偏高, 距离南淝河越近越突出 ${ }^{[21-22]}$. 这是由于南淝河与塘西河靠近合肥市, 人口 密集, 此外受夏季东南风及水动力的影响, 南淝河人湖口附近为蓝藻水华聚集地, 水华蓝藻生物量大且吸附 重金属多,死亡后释放重金属, 导致重金属在湖泊内显著迁移. 李国莲等 ${ }^{[23]}$ 的研究指出巢湖西半湖南淝河 口水体因受到合肥市工业与生活污染排放的直接影响, Cd 含量最大值为 $0.07 \mu \mathrm{g} / \mathrm{L}$. 本研究中, 巢湖水华蓝 藻中的 $\mathrm{Cd}$ 含量为 $0.1 \mathrm{mg} / \mathrm{kg}$. 藻类体内的微量元素主要源自对水体、沉积物中微量元素的吸收和富集, 来源 有工业生产、农业活动以及城乡生活等. 由此可知, 巢湖西半湖岸沉积物重金属除了合肥市的废水污染源 外 ${ }^{[24]}$,部分来源于蓝藻水华集聚与腐殖化过程对重金属的迁移与释放作用.

表 1 巢湖沉积物中重金属含量

Tab.1 Heavy metal contents in sediments of Lake Chaohu

\begin{tabular}{|c|c|c|c|c|c|c|c|}
\hline 采样点 & $\begin{array}{c}\mathrm{Cd} / \\
(\mathrm{mg} / \mathrm{kg})\end{array}$ & $\begin{array}{c}\mathrm{Cr} / \\
(\mathrm{mg} / \mathrm{kg})\end{array}$ & $\begin{array}{c}\mathrm{Cu} / \\
(\mathrm{mg} / \mathrm{kg})\end{array}$ & $\begin{array}{c}\mathrm{Mn} / \\
(\mathrm{mg} / \mathrm{kg})\end{array}$ & $\begin{array}{c}\mathrm{Ni} / \\
(\mathrm{mg} / \mathrm{kg})\end{array}$ & $\begin{array}{c}\mathrm{Pb} / \\
(\mathrm{mg} / \mathrm{kg})\end{array}$ & $\begin{array}{c}\mathrm{Zn} / \\
(\mathrm{mg} / \mathrm{kg})\end{array}$ \\
\hline 背景值 * & 0.097 & 66.5 & 20.4 & 634 & 29.8 & 26.6 & 62 \\
\hline 南淝河人湖口 & $\begin{array}{c}0.08 \sim 0.75 \\
(0.389)\end{array}$ & $\begin{array}{c}65.63 \sim 112.1 \\
(85.380)\end{array}$ & $\begin{array}{c}13.27 \sim 41.4 \\
(29.37)\end{array}$ & $\begin{array}{c}513.6 \sim 1561 \\
(1088.66)\end{array}$ & $\begin{array}{c}16.69 \sim 41.71 \\
(28.12)\end{array}$ & $\begin{array}{c}27.9 \sim 64.94 \\
(45.44)\end{array}$ & $\begin{array}{c}192.6 \sim 589.4 \\
(300.6)\end{array}$ \\
\hline 杭埠河人湖口 & $\begin{array}{c}0.196 \sim 0.32 \\
\quad(0.26)\end{array}$ & $\begin{array}{c}48.51 \sim 106.9 \\
\quad(82.73)\end{array}$ & $\begin{array}{c}4.57 \sim 22.29 \\
\quad(12.20)\end{array}$ & $\begin{array}{l}760 \sim 1700 \\
(1221.75)\end{array}$ & $\begin{array}{c}13.47 \sim 29.97 \\
(21.20)\end{array}$ & $\begin{array}{c}16.61 \sim 27.96 \\
(23.76)\end{array}$ & $\begin{array}{c}60.38 \sim 120.1 \\
\quad(90.05)\end{array}$ \\
\hline 上派河人湖口 & $\begin{array}{c}0.1 \sim 0.11 \\
\quad(0.06)\end{array}$ & $\begin{array}{c}53.05 \sim 71.25 \\
(63.38)\end{array}$ & $\begin{array}{c}9.07 \sim 14.29 \\
\quad(11.55)\end{array}$ & $\begin{array}{c}339.1 \sim 612.4 \\
(516.53)\end{array}$ & $\begin{array}{c}14.18 \sim 16.66 \\
(15.42)\end{array}$ & $\begin{array}{c}15.63 \sim 24.76 \\
(19.97)\end{array}$ & $\begin{array}{c}52.06 \sim 110.3 \\
\quad(79.23)\end{array}$ \\
\hline 塘西河人湖口 & $\begin{array}{c}0.53 \sim 0.59 \\
\quad(0.56)\end{array}$ & $\begin{array}{c}72.77 \sim 109.8 \\
(91.29)\end{array}$ & $\begin{array}{c}30.39 \sim 31.52 \\
(30.96)\end{array}$ & $\begin{array}{c}1417 \sim 1425 \\
\quad(1421)\end{array}$ & $\begin{array}{c}41.47 \sim 42.9 \\
\quad(42.19)\end{array}$ & $\begin{array}{c}56.59 \sim 65.26 \\
(60.93)\end{array}$ & $\begin{array}{c}333.6 \sim 409.4 \\
(371.5)\end{array}$ \\
\hline 西半湖湖心 & $\begin{array}{c}0.11 \sim 0.75 \\
\quad(0.42)\end{array}$ & $\begin{array}{c}77.9 \sim 151.3 \\
(112.7)\end{array}$ & $\begin{array}{c}21.12 \sim 36.94 \\
\quad(28.7)\end{array}$ & $\begin{array}{c}933.9 \sim 1942 \\
(1455.5)\end{array}$ & $\begin{array}{c}28.08 \sim 50.83 \\
\quad(40.06)\end{array}$ & $\begin{array}{c}37.71 \sim 73.99 \\
\quad(54.63)\end{array}$ & $\begin{array}{c}173.3 \sim 416.2 \\
(271.22)\end{array}$ \\
\hline
\end{tabular}

* 数据来源于安徽省土壤重金属环境背景值; 括号内数据为河口重金属含量均值.

\section{2 表层沉积物中重金属形态分布特征及各形态之间的相关性}

巢湖表层沉积物中重金属 Mn 和 Cd 的 F1 态为其主要赋存形态, 平均含量可达 $53.24 \%$ 和 $45.69 \%, \mathrm{Cd}$ 其 次为 $\mathrm{F} 2$ 态, 平均含量为 $23.76 \%, \mathrm{Mn}$ 的 $\mathrm{F} 2$ 和 $\mathrm{F} 4$ 态含量较高, 平均为 $20.55 \%$ 和 $27.06 \%$ (图 3). $\mathrm{Cd}$ 易与 $\mathrm{Ca}^{2+}$ 发生替代反应, 因此造成沉积物中 $\mathrm{Cd}$ 的 F1 态较高 ${ }^{[25]}$. F1 态的重金属对 $\mathrm{pH}$ 值的敏感度较高, 在酸性条件下 容易释放,夏季蓝藻腐殖化过程会导致水体 $\mathrm{pH}$ 和溶解氧浓度下降 ${ }^{[4,26]}$, 弱酸提取态较容易被释放出来, 同 时弱酸提取态会被还原成生物可利用态并由沉积物向水体释放, 能被植物吸收. 而残渣态比例较高时, 在水 体理化性质改变时, 这部分赋存在原生矿物和次生硅酸盐矿物晶格中的重金属释放到水体, 造成水体二次 污染,潜在生物迁徙性较大.

由表 2 可知, 巢湖西半湖、南淝河人湖口、杭埠河人湖口以及上派河人湖口沉积物中 $\mathrm{Cd}$ 的 $P M I$ 均值分 别为 $76.9 \% 、 74.9 \% 、 83.8 \%$ 和 $84.7 \%$, 各区域均值都较高, 但南汁河的最高值 $(94.8 \%)$ 明显高于杭埠河 $(89.5 \%)$ 及上派河 $(88.5 \%)$, 说明整个西半湖 $\mathrm{Cd}$ 的迁移性较大; 西半湖、南淝河人湖口、杭埠河人湖口以及 上派河人湖口沉积物中 $\mathrm{Mn}$ 的 $P M I$ 均值分别为 $64.5 \% 、 67.7 \% 、 63.1 \%$ 和 $60.2 \%$, 说明整个西半湖沉积物中 $\mathrm{Mn}$ 的迁移性相差不大. 各采样点 $\mathrm{Cd}$ 的 $P M I$ 均超出 $\mathrm{Mn}$, 表明 $\mathrm{Cd}$ 比 Mn 更易形成二次污染. 且西半湖沿岸蓝藻 积聚, 藻类生长影响了界面 $\mathrm{pH} 、 \mathrm{DO}$ 浓度等水体环境, 提高了 $\mathrm{Cd}$ 从沉积物内部向水体系中扩散的速率. 因此 在藻类生长、迁移和死亡过程中藻细胞可能会吸附积累及释放重金属, 从而影响到重金属在水体中的含量 分布及形态变化 ${ }^{[27]}$. 


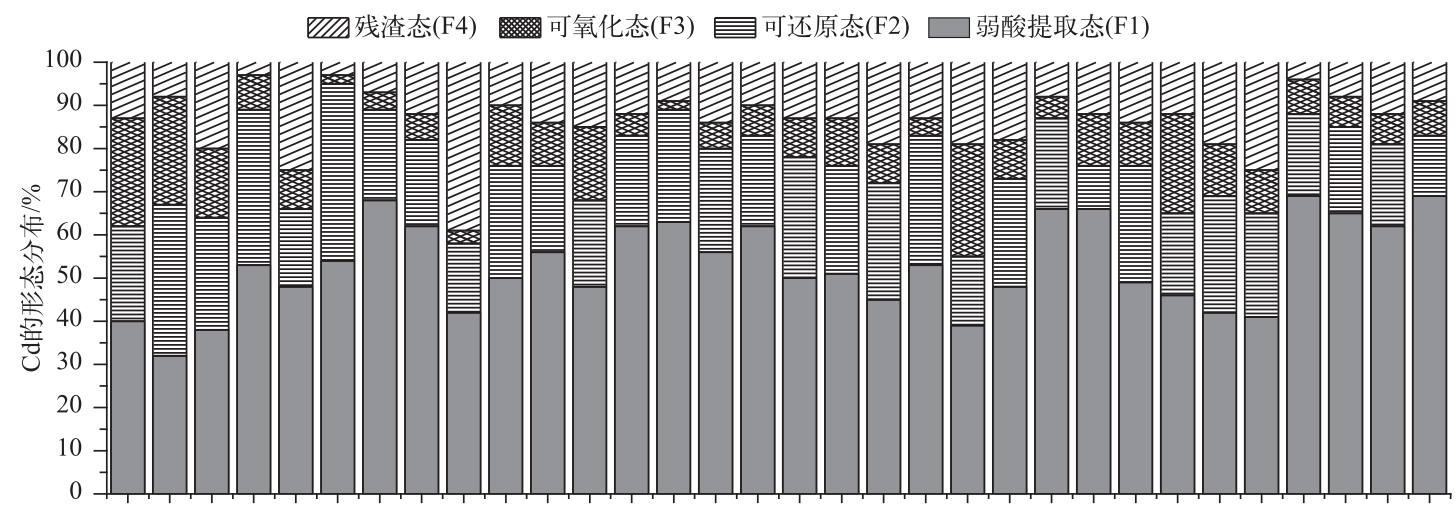

N0 N1 N2 N3 N4 N5 N6 N7 N8N9N10N11 H0 H1 H4 H6 X1 X3 X4 X5 X6 X7 X8X10X11W2 T2 T3 S1 S3 S4 B1

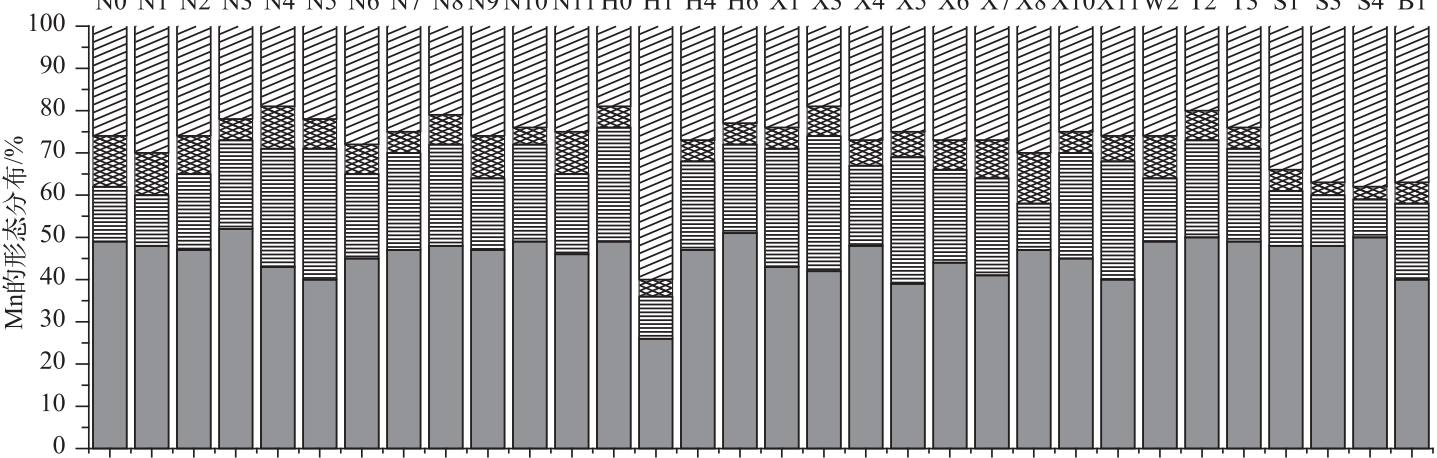

N0 N1 N2 N3 N4 N5 N6 N7 N8 N9 N10 N11 H0 H1 H4 H6 X1 X3 X4 X5 X6 X7 X8 X10X11W2 T2 T3 S1 S3 S4 B1 采样点

图 3 巢湖各采样点不同形态重金属所占百分比

Fig.3 Percentage of different factions of heavy metals for different sampling sites of Lake Chaohu

表 2 巢湖表层沉积物中重金属潜在迁移指数 $(P M I) *$

Tab.2 PMI of heavy metals in surface sediments of Lake Chaohu

\begin{tabular}{ccccc}
\hline 重金属 & 南淝河人湖口/\% & 杭埠河人湖口/\% & 上派河人湖口 $/ \%$ & 西半湖 $/ \%$ \\
\hline \multirow{2}{*}{$\mathrm{Cd}$} & $58.1 \sim 94.8$ & $80.3 \sim 89.5$ & $81.2 \sim 88.5$ & $72.1 \sim 87.4$ \\
& $(74.9)$ & $(83.8)$ & $(84.7)$ & $(76.9)$ \\
$\mathrm{Mn}$ & $60.1 \sim 73.2$ & $36.3 \sim 76.2$ & $59.1 \sim 61.5$ & $(60.2)$ \\
& $(67.7)$ & $(63.1)$ & $(64.5)$ \\
\hline
\end{tabular}

* 括号内数据为各采样点 $P M I$ 均值.

由相关性分析 (表 3) 可知,除 $\mathrm{Mn}$ 的残渣态含量与总量之间表现出显著正相关 $(P<0.05)$ 外, $\mathrm{Mn}$ 和 $\mathrm{Cd}$ 的各形态含量和总量之间均表现出极显著正相关 $(P<0.01)$, 同时, 这两种重金属的 F1 态与 F2 及 F3 态含量 之间表现出极显著正相关 $(P<0.01)$. Cd 的 F4 态含量与其他形态含量之间均存在极显著正相关 $(P<0.01)$, 表明残渣态的稳定性是相对的, 在蓝藻水华腐殖化过程中, 残渣态则可能被释放到水体.

作为可以比较准确判断沉积物有机质来源的指标, TOC/TN 比值可以有效区分沉积有机质的藻类和陆 生植物来源. 重金属 $\mathrm{Cd}$ 和 $\mathrm{Mn}$ 的不同形态含量和总量之间均表现出极显著正相关, 其中 $\mathrm{Mn}$ 的残渣态含量 和总量之间表现出显著正相关 (表 3), 表明重金属不同形态的来源具有一致性并且分布类型比较相似. 本 文研究发现, 巢湖南淝河附近及西部湖心区 TOC/TN 比值均介于 $2.03 \sim 4.17$ 之间, 属于典型的藻型湖 ${ }^{[28]}$, 周 志华等通过研究判定巢湖西半湖沉积物的物质来源主要是湖泊自身 ${ }^{[29]}$, 表明巢湖沉积物有机质来源于蓝 
藻. Cd 的不同形态含量和总量都与 TN、TOC 含量存在显著正相关, Mn 除残渣态以外, 其他形态含量和总量 均与 TOC、TN 含量表现出极显著正相关和显著正相关 (表 3), 表明 Cd、Mn 来源和分布与蓝藻有关, 蓝藻水 华腐殖化过程可能会对水体、沉积物重金属有吸附和释放的影响.

表 3 巢湖沉积物中不同形态重金属含量与其他指标之间的相关性分析

Tab.3 Correlations analyses between different heavy metal contents and other indicators in the sediments of Lake Chaohu

\begin{tabular}{|c|c|c|c|c|c|c|c|c|}
\hline 重金属 & 指标 & $\mathrm{TN}$ & TOC & $\begin{array}{c}\text { 弱酸提 } \\
\text { 取态 }(\mathrm{F} 1)\end{array}$ & $\begin{array}{l}\text { 可还原 } \\
\text { 态 (F2) }\end{array}$ & $\begin{array}{l}\text { 可氧化 } \\
\text { 态 (F3) }\end{array}$ & $\begin{array}{c}\text { 残渣态 } \\
\text { (F4) }\end{array}$ & 总量 \\
\hline \multirow[t]{7}{*}{ Cd } & $\mathrm{TN}$ & 1.000 & & & & & & \\
\hline & TOC & & 1.000 & & & & & \\
\hline & 弱酸提取态 (F1) & $0.586^{* *}$ & $0.592^{* *}$ & 1.000 & & & & \\
\hline & 可还原态 (F2) & $0.518^{* * *}$ & $0.633^{*}$ & $0.870^{* * *}$ & 1.000 & & & \\
\hline & 可氧化态 (F3) & $0.315^{*}$ & $0.440^{\text {*** }}$ & $0.506^{* *}$ & $0.547^{* * *}$ & 1.000 & & \\
\hline & 残渣态( F4) & $0.649^{* *}$ & $0.496^{* *}$ & $0.542^{* *}$ & $0.423 *$ & $0.457^{* * *}$ & 1.000 & \\
\hline & 总量 & $0.736^{* *}$ & $0.725^{\text {** }}$ & $0.844^{* *}$ & $0.825^{* *}$ & $0.609^{* * *}$ & $0.639^{* * *}$ & 1.000 \\
\hline \multirow[t]{7}{*}{ Mn } & $\mathrm{TN}$ & 1.000 & & & & & & \\
\hline & TOC & & 1.000 & & & & & \\
\hline & 弱酸提取态 (F1) & $0.735^{* *}$ & $0.577^{* *}$ & 1.000 & & & & \\
\hline & 可还原态 (F2) & 0.481 ** & $0.382^{*}$ & $0.675^{* *}$ & 1.000 & & & \\
\hline & 可氧化态 (F3) & $0.733 * *$ & $0.676^{* *}$ & $0.669^{* *}$ & $0.511^{* * *}$ & 1.000 & & \\
\hline & 残渣态( F4) & 0.006 & 0.065 & 0.164 & 0.204 & 0.220 & 1.000 & \\
\hline & 总量 & $0.732 * *$ & $0.559^{* *}$ & $0.815^{* *}$ & $0.525^{* * *}$ & $0.762^{* *}$ & $0.400^{*}$ & 1.000 \\
\hline
\end{tabular}

** 表示在 $P<0.01$ 时极显著相关 (双尾检验) ; * 表示在 $P<0.05$ 时显著相关 (双尾检验) $; n=32$.

\section{3 蓝藻腐殖化过程}

2.3.1 蓝藻腐殖化过程中水体 $\mathrm{pH}$ 的变化 蓝藻腐殖化 过程使水体 $\mathrm{pH}$ 发生了显著的变化 (图 4). 藻类的代谢 随光线变化而变化,光照下,光合作用占优势, 吸收水体 中的二氧化碳,避光处理, 呼吸作用向水体释放二氧化 碳. $\mathrm{A}$ 组与 B 组前 $30 \mathrm{~d}$, 因实验环境与外界隔离,无外来 营养源,光合作用与呼吸作用平衡,两组 $\mathrm{pH}$ 变化不大, 且变化较为一致. $30 \mathrm{~d}$ 以后, $\mathrm{A}$ 组呈上升趋势, 可能是由 于前 $30 \mathrm{~d}$ 蓝藻消耗体内营养物质较多,后期光合作用强 度高于呼吸强度,消耗水体 $\mathrm{CO}_{2}$, 导致水体 $\mathrm{pH}$ 升高; 而 $B$ 组第 $30 \mathrm{~d}$ 后与 $\mathrm{C}$ 组整个阶段均进人厌氧阶段, 由于细 胞内的聚磷酸体的消耗 ${ }^{[30]}$, 同时避光处理, 呼吸作用强 于光合作用,产生 $\mathrm{CO}_{2}$, 导致水体 $\mathrm{pH}$ 持续下降; 且 $\mathrm{C}$ 组 整个阶段厌氧避光, 蓝藻腐殖化过程中, 元素碳生成 $\mathrm{CH}_{4}$ 和 $\mathrm{CO}_{2}{ }^{[31]}$, 同时呼吸作用较强, 使得实验整个阶段

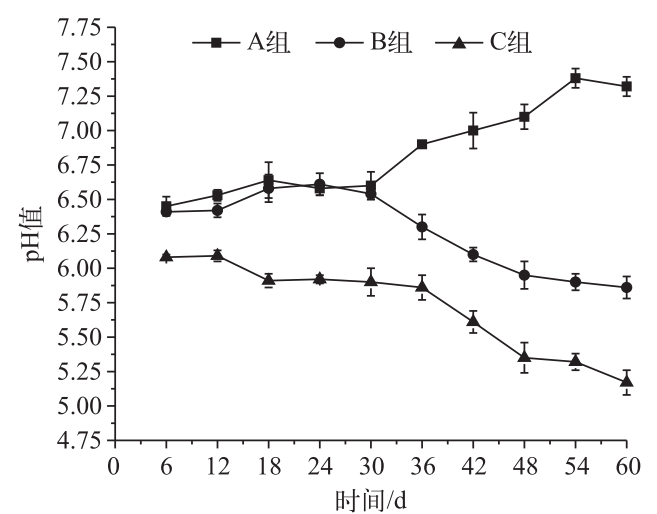

图 4 上覆水 $\mathrm{pH}$ 值随时间的变化曲线

Fig.4 The curve of $\mathrm{pH}$ value of overlying water with the change of time $\mathrm{pH}$ 值低于 $\mathrm{A}$ 组和 $\mathrm{B}$ 组.

2.3.2 蓝藻腐殖化过程中藻体及上覆水中重金属含量变化 好氧过程中, 藻体中重金属含量大致呈波动性上 升趋势, 上覆水中重金属含量呈波动性下降趋势. 而 $\mathrm{B}$ 组上覆水中 $\mathrm{Cd}$ 含量则出现了先增加后减少的变化, 这可能是由于蓝藻对 $\mathrm{Cd}$ 的吸附最适 $\mathrm{pH}$ 为 $6^{[32]}, \mathrm{pH}$ 偏高会影响蓝藻对重金属的吸附效率. 好氧阶段前期可 能由于 $\mathrm{pH}$ 的升高影响到重金属离子的解离状态, 进而对重金属的吸附作用产生影响, 导致 $\mathrm{A}$ 组和 $\mathrm{B}$ 组上覆 水中 $\mathrm{Cd}$ 含量先增加后减少 (图 5). 

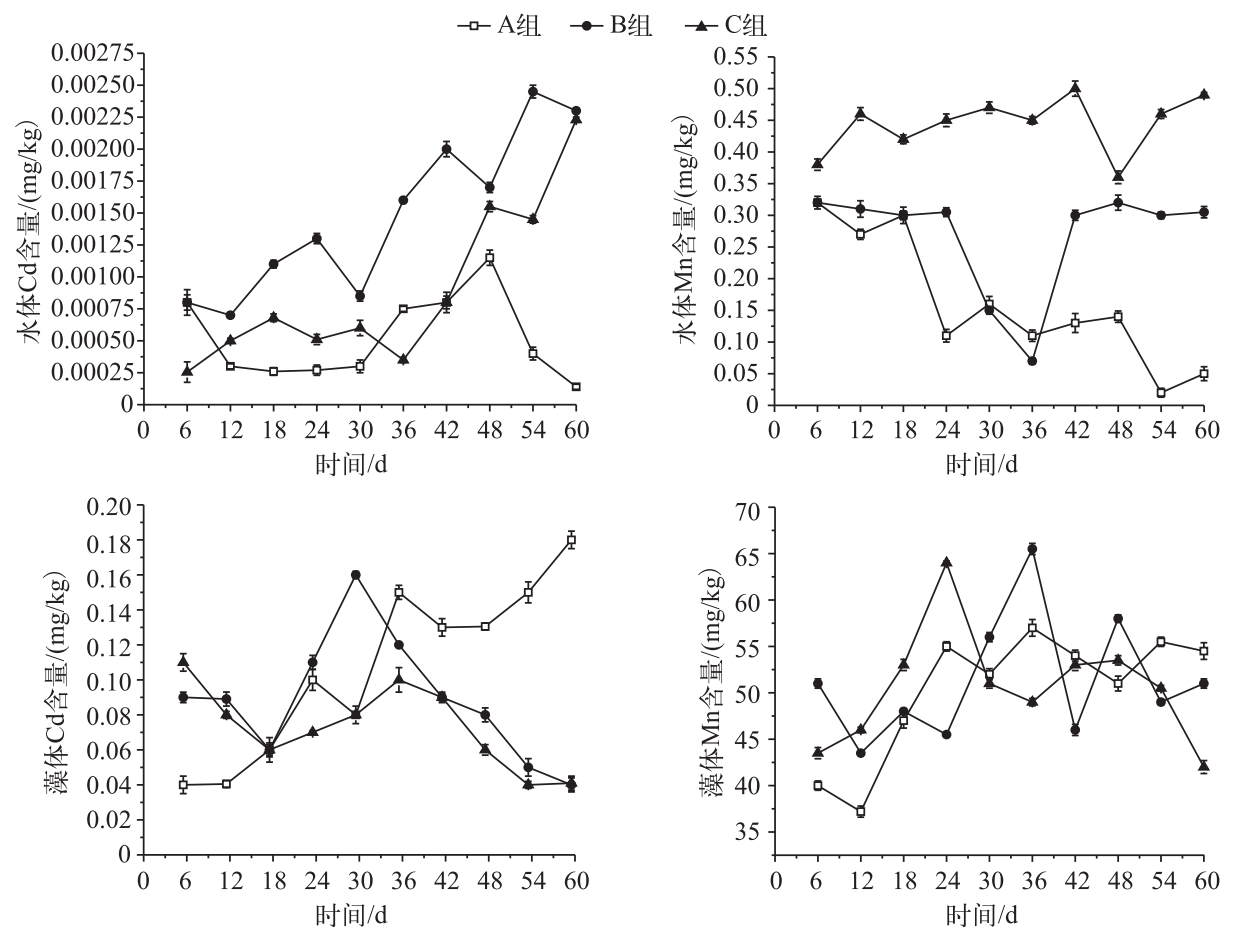

图 5 蓝藻腐殖化过程中藻体和水体重金属含量随时间的变化曲线

Fig.5 The curve of heavy metal content in alga and water with the change of time during the process of the humification of blue-green algae

厌氧阶段蓝藻细胞裂解, 与蓝藻胞内蛋白质、核酸等可溶性有机物结合的重金属会被释放至上覆水. 由 图 5 可以看出, 藻体重金属含量呈波动性下降趋势, 上覆水中重金属含量则大致表现为上升趋势, 与理论结 果相一致. 其中 $\mathrm{C}$ 组藻体中 $\mathrm{Cd}$ 和 $\mathrm{Mn}$ 含量表现出先上升后下降的曲线, $\mathrm{C}$ 组上覆水中 $\mathrm{Mn}$ 含量后期出现降 低且波动幅度较大, 可能是由于死藻细胞壁破碎较多, 细胞表面暴露的官能团对上覆水中重金属同样有吸 附作用所致 ${ }^{[33]}$. 后由于微生物的作用, 蓝藻残渣被分解, 重金属又被重新释放回水体, 导致上覆水中 $\mathrm{Cd}$ 和 Mn 含量的增加.

通过模拟实验可以发现, 蓝藻腐殖化过程会导致水体 $\mathrm{pH}$ 的变化, 影响到重金属离子的解离状态, 同时 腐殖化过程中蓝藻会对上覆水重金属产生吸附-释放、再吸附-释放作用.

\section{3 结论}

1) 巢湖西半湖表层沉积物中重金属 $(\mathrm{Cd} 、 \mathrm{Cr} 、 \mathrm{Cu} 、 \mathrm{Zn} 、 \mathrm{~Pb} 、 \mathrm{Ni}$ 和 $\mathrm{Mn})$ 分布具有明显的空间差异性, 靠近南 淝河, 超出背景值较明显, 表明巢湖西半湖岸沉积物中重金属除了来源于合肥市废水, 可能部分来源于蓝藻 水华集聚与腐殖化过程对重金属的迁移与释放作用.

2) $P M I$ 计算表明重金属 $\mathrm{Cd}$ 比 $\mathrm{Mn}$ 更易迁移, $\mathrm{Mn}$ 和 $\mathrm{Cd}$ 各形态含量和总量之间均表现出极显著正相关, 同时这两种重金属的弱酸提取态与可还原态及可氧化态之间表现出极显著正相关,表明这两种重金属的来 源和分布具有一致性.

$3)$ 模拟实验发现, 蓝藻腐殖化过程会导致水体 $\mathrm{pH}$ 的变化, 影响到重金属离子的解离状态, 同时腐殖化 过程中蓝藻会对上覆水重金属产生吸附-释放、再吸附-释放作用.

\section{4 参考文献}

[ 1 ] Ciutat A, Boudou A. Bioturbation effects on cadmium and zinc transfers from a contaminated sediment and on metal bio- 
availability to benthic bivalves. Environmental Toxicology \& Chemistry, 2003, 22(7) : 1574-1581.

[ 2 ] Yu Xiujuan, Huo Shouliang, Zan Fengyu et al. Distribution characteristics and contamination assessment of heavy metals in surface sediments of Lake Chaohu, China. Chinese Journal of Environmental Engineering, 2013, 7(2) : 439-450. [余秀 娟, 霍守亮, 处逢宇等. 巢湖表层沉积物中重金属的分布特征及其污染评价. 环境工程学报, 2013, 7(2): 439-450.]

[ 3 ] Song Xianqiang, Lei Hengyi, Yu Guangwei et al. Evaluation of heavy metal pollution and release from sediment in a heavily polluted tidal river. Acta Science Circumstantiae, 2008, 28(11): 2258-2268. [宋宪强, 雷恒毅, 余光伟等. 重污染 感潮河道底泥重金属污染评价及释放规律研究. 环境科学学报, 2008, 28(11): 2258-2268.]

[ 4 ] Ni Lixiao, Ye Xiang, Ma Yanyan et al. Effects of cyanophytes on the migration-release of cadmium in sediment by laboratorial microcosm. Guangdong Agricultural Science, 2013, 40(3): 147-150, 154. [倪利晓, 叶祥, 马艳艳等. 室内模拟 水华蓝藻对沉积物中 Cd 迁移释放的影响. 广东农业科学, 2013, 40(3) : 147-150, 154.]

[ 5 ] Li Yinxia, Rao Benqiang, Wang Zhicong et al. Spatial-temporal distribution of phytoplankton in bloom-accumulation area in Lake Chaohu. Resources and Environment in the Yangtze Basin, 2012, (S2): 25-31. [李印霞, 饶本强, 汪志聪等. 巢湖藻华易堆积区蓝藻时空分布的研究. 长江流域资源与环境, 2012, (S2) : 25-31.]

[ 6 ] Kong Ming, Zhang Lu, Yin Hongbin et al. Influence of algae bloom on distribution of total and speciation of nitrogen and phosphorus in the surface sediments from Lake Chaohu. China Environmental Science, 2014, 34(5):1285-1292. [孔明, 张路, 尹洪斌等. 蓝藻暴发对巢湖表层沉积物氮磷及形态分布的影响. 中国环境科学, 2014, 34(5): 1285-1292.]

[ 7 ] Zhang Min, Kong Fanxiang. The process spatial and temporal distributions and mitigation strategies of the eutrophication of Lake Chaohu(1984-2013). J Lake Sci, 2015, 27(5) : 791-798. DOI:10.18307/2015.0505. [张民, 孔繁翔. 巢湖富营 养化的历程、空间分布与治理策略(1984-2013 年). 湖泊科学, 2015, 27(5): 791-798.]

[ 8 ] Yao Shuchun, Li Shijie. Sedimentary records of eutrophication for the last 100 years in Lake Chaohu. Acta Sedimentologica Sinica, 2004, 22(2) : 343-347. [姚书春, 李世杰. 巢湖富营养化过程的沉积记录. 沉积学报, 2004, 22(2): 343-347.]

[ 9 ] Jia Tiefei, Zhang Weiguo, Yu Lizhong. Metal element enrichment characteristics of sediments in Lake Chaohu since the 1860s and its implication to human activity. Geographical Research, 2009, (5): 1217-1226. [贾铁飞, 张卫国, 俞立中. $1860 \mathrm{~s}$ 以来巢湖沉积物金属元素富集特点及其对人类活动的指示意义. 地理研究, 2009, (5): 1217-1226.]

[10] Zheng Zhixia, Pan Chengrong, Ding Fan. Distribution and environmental pollution assessment of heavy metals in surface sediments of Lake Chaohu, China. Journal of Agro-Environment Science, 2011, 30(1): 161-165. [郑志侠, 潘成荣, 丁 凡. 巢湖表层沉积物中重金属的分布及污染评价. 农业环境科学学报, 2011, 30(1): 161-165.]

[11] Kong Ming, Dong Zenglin, Chao Jianying et al. Bioavailability and ecological risk assessment of heavy metals in surface sediments of Lake Chaohu. China Environmental Science, 2015, 35(4): 1223-1229. [孔明, 董增林, 晁建顷等. 巢湖 表层沉积物重金属生物有效性与生态风险评价. 中国环境科学, 2015, 35(4) : 1223-1229.]

[12] Chen Sijia, Zheng Wenjie, Yang Fang. Study advances on heavy metals bio-absorbed by cyanobacteria. Marine Environmental Science, 2006, 25(4) : 103-106. [陈思嘉, 郑文杰, 杨芳. 蓝藻对重金属的生物吸附研究进展. 海洋环境科 学, 2006, 25(4): 103-106.]

[13] Li PF, Mao ZY, Rao XJ et al. Biosorption of uranium by lake-harvested biomass from a cyanobacterium bloom. Bioresour Technol, 2004, 94(2) : 193-195.

[14] Singh S, Pradhan S, Rai LC. Comparative assessment of $\mathrm{Fe}^{3+}$ and $\mathrm{Cu}^{2+}$ biosorption by field and laboratory-grown Microcystis. Process Biochemistry, 1998, 33(5): 495-504.

[15] Rangsayatorn N, Upatham ES, Kruatrachue $M$ et al. Phytoremediation potential of Spirulina (Arthrospira) platensis: Biosorption and toxicity studies of cadmium. Environmental Pollution, 2002, 119(1) : 45-53.

[16] Wu Wenjuan, Li Jianhong, Liu Chang et al. Reuse of Microcystis bloom: An experimental study on the adsorption of heavy metals $\mathrm{Cu}^{2+}, \mathrm{Cd}^{2+}$ and $\mathrm{Ni}^{2+}$ by bloom biomass. J Lake Sci, 2014, 26(3) : 417-422. DOI: 10.18307/2014.0312. [吴文 娟, 李建宏, 刘畅等. 微囊藻水华的资源化利用: 吸附重金属离子 $\mathrm{Cu}^{2+} 、 \mathrm{Cd}^{2+}$ 和 $\mathrm{Ni}^{2+}$ 的实验研究. 湖泊科学, 2014, 26(3) : 417-422.]

[17] Jiang Jing, Li Liang, Li Haipeng et al. Biosorption of lead ( II ) and cadmium ( II ) from aqueous solution by Chlorella pyrenoidsa and its influential factors. Acta Ecologica Sinica, 2012, 32(7): 1995-2003. [姜晶, 李亮, 李海鹏等. 蛋白核 小球藻对 $\mathrm{Pb}(\mathrm{II})$ 和 $\mathrm{Cd}$ ( II ) 的生物吸附及其影响因素. 生态学报, 2012, 32(7) : 1995-2003. ] 
[18] Li Xiaochen. Distribution of chemical fractions and potential mobility of heavy metals during bio-treatment process of municipal wastewater[Dissertation]. Nanjing: Hohai University, 2006. [李晓晨. 城市污水处理过程中重金属形态分布及 潜在迁移性研究 [学位论文]. 南京: 河海大学, 2006.]

[19] Chen M, Li XM, Yang Q et al. Total concentrations and speciation of heavy metals in municipal sludge from Changsha, Zhuzhou and Xiangtan in middle-south region of China. Journal of Hazardous Materials, 2008, 160(2) : 324-329.

[20] Liu Xin, Jiang Yu, Gao Junfeng et al. Pollution characteristics of heavy metals and the risk assessment for the surface sediments from Lake Chaohu and its main tributary rivers. J Lake Sci, 2016, 28(3) : 502-512. DOI: 10.18307/2016.0305. [刘新, 蒋豫, 高俊峰等. 巢湖湖区及主要出人湖河流表层沉积物重金属污染特征及风险评价. 湖泊科学, 2016, 28(3): 502-512.]

[21] Yin HB, Deng JC, Shao SG et al. Distribution characteristics and toxicity assessment of heavy metals in the sediments of Lake Chaohu, China. Environmental Monitoring and Assessment, 2011, 179(1/2/3/4): 431-442.

[22] Liu Cheng, Shao Shiguang, Fan Chengxin et al. Pollution status and risk assessment of heavy metal in the sediment of the severe polluted confluence area of Lake Chaohu. China Environmental Science, 2014, 34(4): 1031-1037. [刘成, 邵世 光, 范成新等. 巢湖重污染汇流湾区沉积物重金属污染特征及风险评价. 中国环境科学, 2014, 34 (4): 1031-1037.]

[23] Li GuoLian, Liu Guijian, Jiang Mengmeng et al. Partition characteristics and correlation of heavy metal between sediment and surface water from Lake Chaohu. Journal of University of Science and Technology of China, 2011, 41(1): 9-15. [李 国莲, 刘桂建, 姜萌萌等. 巢湖表层沉积物与上覆水体中重金属分配特征及其相关性研究. 中国科学技术大学学 报, 2011, 41(1): 9-15.]

[24] Du Chenchang, Liu Enfeng, Yang Xiangdong et al. Characteristics of enrichment and evaluation of anthropogenic pollution of heavy metals in the sediments of Lake Chaohu. J Lake Sci, 2012, 24(1) : 59-66. DOI: 10.18307/2012.0108. [ 杜臣 昌, 刘恩峰, 羊向东等. 巢湖沉积物重金属富集特征与人为污染评价. 湖泊科学, 2012, 24(1): 59-66.]

[25] Liu Enfeng, Shen Ji, Zhu Yuxin. Determination of heavy metal chemical forms by ber method for Taihu Lake sediments. Research of Environmental Sciences, 2005,18(2): 57-60. [刘恩峰, 沈吉, 朱育新. 重金属元素 BCR 提取法及在太湖 沉积物研究中的应用. 环境科学研究, 2005, 18(2): 57-60.]

[26] Shang Lixia, Ke Fan, Li Wenchao et al. Laboratory research on the contaminants release during the anaerobic decomposition of high-density cyanobacteria. J Lake Sci, 2013, 25(1) : 47-54. DOI:10.18307/2013.0107. [尚丽霞, 柯凡, 李文 朝等. 高密度蓝藻厌氧分解过程与污染物释放实验研究. 湖泊科学, 2013, 25(1): 47-54.]

[27] Ni Lixiao, Ma Yanyan, Ye Xiang et al. Study on Biosorption of cadmium by Microcystis Aeruginosa at different cell activity and nutrient level. Environmental Science and Technology, 2013, 26(3): 1-4. [倪利晓, 马艳艳, 叶祥等. 藻细胞活性 及营养盐水平对铜绿微囊藻吸附镉的影响研究. 环境科技, 2013, 26(3) : 1-4.]

[28] Meyers PA. Preservation of elemental and isotopic source identification of sedimentary organic matter. Chemical Geology, 1994, 114(3/4): 289-302.

[29] Zhou Zhihua, Liu Congqiang, Li Jun et al. Record of ecosystem evolvement processes provided by $\delta^{13} \mathrm{C}_{\text {org }}$ and $\delta^{15} \mathrm{~N}$ values in Lake Chaohu sediments. Environmental Science, 2007, 28(6): 1338-1343, 1343. [周志华, 刘丛强, 李军等. 巢湖 沉积物 $\delta^{13} \mathrm{C}(\mathrm{org})$ 和 $\delta^{15} \mathrm{~N}$ 记录的生态环境演化过程. 环境科学, 2007, 28 (6) : 1338-1343, 1343.]

[30] Li Jie, Ou Danyun, Song Lirong. Decline of Microcystis aeruginosa FACHB-905 under four stress conditions. J Lake Sci, $2008,20(5)$ : 549-555. DOI: 10.18307/2008.0501. [李杰, 欧丹云, 宋立荣. 微囊藻衰亡过程研究一一四种模拟胁 迫条件下微囊藻的衰亡生理. 湖泊科学, 2008, 20(5): 549-555.]

[31] Li Yonghui, Li Yucheng, Wang Ning et al. Formation and control of the harmful ingredients during the decaying process of cyanobacteria from Lake Chaohu. J Lake Sci, 2012, 24(4) : 513-518. DOI: 10.18307/2012.0402. [李永慧, 李玉成, 王 宁等. 巢湖蓝藻死亡衰败过程中典型有害成分形成及控制对策. 湖泊科学, 2012, 24(4): 513-518.]

[32] Romera E, González F, Ballester A et al. Comparative study of biosorption of heavy metals using different types of algae. Bioresource Technology, 2007, 98(17) : 3344-3353.

[33] Costa ACAD, França FPD. Cadmium uptake by Spirulina maxima: Toxicity and mechanism. World Journal of Microbiology \& Biotechnology, 1998, 14(14): 579-581. 